\title{
Proyectos Fotovoltaicos en Generación Distribuida ¿Rentabilidad Privada o Sustentabilidad Ambiental?
}

\author{
Cisterna Arellano, Luis 1 (D); Améstica-Rivas, Luis ${ }^{1}$ * (D); Piderit, María Beatriz ${ }^{2}$ (iD) \\ ${ }^{I}$ Depto. de Gestión Empresarial, Facultad de Ciencias Empresariales, Universidad del Bío-Bío, Chile \\ ${ }^{2}$ Depto. Diseño y Teoría de la Arquitectura, Facultad de Arquitectura, Construcción y Diseño, Universidad del Bío-Bío, Chile
}

\begin{abstract}
Resumen: Las energías renovables son pilares estratégicos para disminuir la emisión de gases y daños a la atmosfera. Se reconoce un crecimiento exponencial, destacando la energía solar a través de instalaciones fotovoltaicas, expresión de ello son las inversiones a escala especialmente en el norte de Chile. En este contexto, este trabajo tiene como objetivo evaluar económicamente proyectos de menor escala como son los de Generación Distribuida, calculando su rentabilidad privada y de carácter social, incluida una valoración económica ambiental de proyectos en la inter comuna de Chillán - Chillán Viejo, como un estudio de caso. Para ello, se analiza la Ley 20.571, que permite a las generadoras residenciales vender sus excedentes de energía a la distribuidora eléctrica a precio regulado. Se realizó un estudio para 17 instalaciones del área residencial, agrícola, industrial y comercial, desarrollando un análisis del Valor Actual Neto (VAN) y la Tasa Interna de Retorno (TIR). Se construyeron los promedios móviles de consumo antes y posterior a sus operaciones, pago directos, costos e ingresos por venta de excedentes. Asimismo, se simuló una compensación de ingresos por venta de bonos de carbono. Los resultados, tanto en evaluación privada como la valoración económica ambiental, arrojan resultados desfavorables, de lo cual se puede inferir que este tipo de proyectos no son rentables en los sectores que abarca el estudio, y es necesario un apoyo estatal a la inversión inicial o la tarifa. Sin embargo, existe una dimensión que va más allá de lo económico que debe sustentar una política fiscal de apoyo para los próximos años.
\end{abstract}

Palabras clave: Energía Solar Fotovoltaica, Generación Distribuida, Rentabilidad, Bonos de carbono.

\section{Photovoltaic Projects in Distributed Generation. Private Profitability or Environmental Sustainability?}

\begin{abstract}
Renewable energies are strategic pillars for reducing gas emissions and atmospheric damage. It is recognized an exponential growth, highlighting solar energy and their photovoltaic installations, a key example of this is the scale of investments, especially in the north of Chile. In this context, this work aims to economically assess smaller-scale projects that are being built, such as those of Distributed Generation, calculating their private and social profitability, including an environmental economic assessment of projects in the Chillán - Chillán Viejo district, as a case study. To do this, Law 20,571 is analyzed. This law allows residential generators to sell their surplus energy to the electricity distributor at a regulated price. A study was made for 17 residential, agricultural, industrial and commercial installations, developing an analysis of the Net Present Value (NPV) and the Internal Rate of Return (IRR).The mobile consumption averages before and after their operations, direct payment, costs and income from the sale of surpluses were built. Likewise, income compensation for the sale of carbon credits was simulated. The results, both in private evaluation and environmental economic assessment, show unfavorable results, and it may be inferred that this type of project is not profitable in the sectors covered by the study, with the need for state support for the initial investment or the tariff. However, there is a dimension that goes beyond the economic that should underpin a supportive fiscal policy for the coming years.
\end{abstract}

Keywords: Photovoltaic Solar Energy, Distributed Generation, Profitability, Carbon credits.

\section{INTRODUCCIÓN}

La transición de las energías desde los combustibles fósiles a la electricidad es una realidad, este hecho está directamente relacionado con el crecimiento económico de un país, así la matriz energética tiende a migrar hacia energías limpias como lo es la electricidad (Jiang \& O'Neill, 2004), esto debido a que la energía eléctrica se puede transformar fácilmente en otras energías como; energía lumínica, energía mecánica, energía térmica, entre otras y de acuerdo con las fuentes de donde se 
obtiene, se pueden diferenciar entre fuentes de energías no renovables y fuentes de energías renovables (ER). Las fuentes de energía no renovables son recursos energéticos que se agotan cuando son empleados, como el petróleo, el gas natural o el carbón y la energía procedente de fuentes renovables se les define como "La energía procedente de fuentes renovables no fósiles, es decir, energía eólica, solar, aerotérmica, geotérmica, hidrotérmica y oceánica, hidráulica, biomasa, gases de vertedero, gases de plantas de depuración y biogás" (Parlamento Europeo y Consejo de la Unión Europea, 2009).

Se ha establecido que la energía solar fotovoltaica (PV) es una solución sostenible para resolver la crisis energética mundial. La problemática se produce en la utilización de fuentes no renovables, como combustibles fósiles o elementos radioactivos, para la generación de energía eléctrica, debido a la notable contribución a la contaminación ambiental, que a su vez está ocasionado un calentamiento global, que está provocando un efecto desbastador sobre el clima de la tierra.

Un estudio señala que por 1 Kilowatts-hora $(\mathrm{kWh})$ gastado en electricidad casera que produce una termoeléctrica, se genera hasta 0,7 kilogramos de CO2 (Medina, 2010). En forma opuesta, el uso de fuentes renovables para producir energía eléctrica minimiza el daño ocasionado al medio ambiente, disminuyendo la producción de gases de efecto invernadero (GEI), debido a que en el proceso no intervienen combustibles fósiles, a estas fuentes de energía que tienen bajo impacto e intervención en el medio, se les conoce como "Energías Renovables No Convencionales" (ERNC) (Behnke et al., 2009), y se les denomina de esta manera, debido a que difiere de otros tipos de energías renovables, en cuanto al uso y cantidad de recursos utilizados.

De las ERNC más conocidas y utilizadas, destaca la energía solar, un estudio realizado el año 2014, hace mención al enorme crecimiento que ha experimentado la energía solar en los últimos 10 años, pasando de un tipo de energía prometedora, a una realidad, debido a que es una energía fácil de producir y totalmente accesible, donde actualmente existen millones de unidades de paneles fotovoltaicos instalados en todo el mundo, cubriendo un área cercana a los 600.000 kilómetros cuadrados (Ovalle Cubillos, 2014). Se destaca a España y Alemania, como los países de la Unión Europea con la más alta capacidad de energía fotovoltaica (FV) instalada, lo que ha generado un aumentado substancialmente las investigaciones en energía solar, la que representan $51 \%$ del total en la UE-27 y existen más publicaciones e investigaciones en este tipo de energía, en comparación con otros tipos de energías renovables, siendo las universidades las que más aportes hacen a esta investigación, en desmedro del bajo interés de la empresa privada. Sin embargo, es el mercado asiático quien domina el mercado global a partir de la aparición de China como el principal mercado FV (Plá, Bolzi \& Durán, 2011). El uso de este tipo de energía ha aumentado más de 20 veces en los últimos 15 años y la meta de la Unión Europea es lograr el $20 \%$ de la matriz energética, este compuesto por este tipo de energías, al año 2020 (Sanz-Casado et al., 2014). Alemania ha sido concebido como líder en la instalación de paneles solares a gran escala sobre las cubiertas de industrias, hogares y granjas, siendo la introducción de la Ley de Tarifas en 1999 la que generó condiciones favorables para los inversores de estos proyectos (Jager-Waldau, 2007).
Otro caso interesante es España, donde se plantea que la evolución de la industria solar permitirá al 2020, que el coste de la electricidad producida mediante fuentes de generación solar sea tan competitivo como el coste de generación de las centrales de ciclo combinado de gas. Se ha hecho mención a la evolución previsible de las tecnologías, también se describe el papel que debería desempeñar, tanto el gobierno como las empresas generadoras, para poder desarrollar un modelo competitivo que permita el desarrollo óptimo de la industria solar en España (González, 2014).

En tanto, en América Latina ha incrementado significativamente su participación en el mercado fotovoltaico a través de la instalación de centrales de potencia como principal fuente (Plá, Bolzi \& Durán, 2011). Son cuatro los países que lideran la generación energía solar: México, Brasil, Argentina y Chile. Este último ha experimentado un crecimiento desde la proclamación de la Ley 20.257 de Energías Renovables No Convencionales del año 2008 (Ministerio de Economía, Fomento y Reconstrucción del Gobierno de Chile, 2008), con una capacidad casi cero en ese año, a más de 1.6 gigavatios en marzo de 2017 (World Economic Forum, 2017). En este contexto, es sabido que los países latinoamericanos son energético-dependientes y esto a su vez refuerza la necesidad de diversificar sus economías y buscar sustitutos energéticos para el futuro (DalmazzoBermejo, Valenzuela-Klagges \& Espinoza-Brito, 2017). En tanto, la realidad energética en Chile señala que el $68 \%$ de la energía eléctrica consumida, es generada por centrales térmicas accionadas por combustibles fósiles, los que deben ser importados en un orden del $90 \%$, esto implica que Chile sea el segundo país, con tarifas eléctricas más altas de Sudamérica, siendo superado sólo por Perú (Rivas y Cardemil, 2015).

Para implementar la generación a través de ERNC en Chile, se han generado marcos legales que incorporan al sistema estas fuentes de generación, así como la forma que se deben compensar. A estas fuentes de generación se les ha denominado Generación Distribuida (GD) y bajo esta denominación se les define como pequeñas fuentes de generación ubicadas en un lugar próximo a los sitios donde es consumida, conectada a la red de distribución de media o baja tensión o directamente a la red pública de distribución (Plá, Bolzi y Durán, 2018; Ackermann, Andersson \& Söde, 2001). Generalmente se asocia al autoabastecimiento (Bermejo y Vasco, 2013). Rüther \& Zilles (2011), establecen que la energía fotovoltaica (FV) a través de sistemas conectados a la red promueve diversos beneficios al sistema eléctrico y el medio ambiente, y como principal ventaja técnica describe la posibilidad de producir electricidad en los propios puntos del consumo.

Tal como se señaló, desde el año 2008 Chile cuenta con la Ley 20.257 (Ministerio de Economía, Fomento y Reconstrucción del Gobierno de Chile, 2008), que promueve la generación de energía eléctrica por medio de fuentes de ERNC. Para ello se eligió el sistema de cuotas, mediante el cual se establece la obligación a las empresas eléctricas, que un porcentaje de la energía que comercializan provenga de fuentes ERNC, específicamente se determina que entre los años 2010 y 2014, la obligación de suministrar energía con medios renovables no convencionales será de 5\%, y a partir de 2015, este porcentaje 
se incrementará en un $0,5 \%$ anual, hasta llegar al $10 \%$ en el año 2024. En la política energética "Energía 2050" (Ministerio de Energía del Gobierno de Chile, 2018b) se define que la ERNC, en 2035 llegue a constituir un $40 \%$ de la generación del país. Una de las grandes deudas de la Ley 20.257, es que no permitía el acceso de las pequeñas instalaciones de ERNC, inyectar los excedentes al sistema eléctrico y con ello, dichos excedentes irremediablemente se perdían al no contar con un sistema de almacenamiento de bajo costo (baterías). Finalmente, este vacío se superó con la Ley 20.571 "Ley de Generación Distribuida" (Ministerio de Energía del Gobierno de Chile, 2012), implementada en septiembre de 2014, esta ley regula el pago de las tarifas eléctricas de las generadoras residenciales y permite la autogeneración de energía en base a ERNC y cogeneración eficiente. Esta ley, conocida también como Generación Distribuida, entrega el derecho a los usuarios a vender sus excedentes directamente a la distribuidora eléctrica a un precio regulado, el cual está publicado en el sitio web de cada empresa distribuidora, cuyo límite de generación actual de 300 Kilowatts (kW).

El crecimiento que ha experimentado las ERNC acogidas a la ley 20.571 ha sido modesto en comparación con la potencia instalada que han alcanzado otros países del año 2012 a la fecha (Salazar-Peralta, Araceli, et al., 2016). Según datos recogidos de la página web www.energiaabierta.cl, del Ministerio de Energía de Chile y como se puede observar en la Figura 1, en el año 2015 se comenzó tímidamente con la puesta en servicio de instalaciones con capacidad de generación de 1.398 Kilowatts (kW) y el año 2017 se llegó a 7.445 kW y al mes de mayo de 2018, se han instalados $5.317 \mathrm{~kW}$, lo que implica que en cuatro años se han incorporado 18.000 Kilowatts $(\mathrm{kW})$ o 18 Megawatts $(\mathrm{mW})$ de potencia instalada de ERNC, a la matriz energética de Chile.

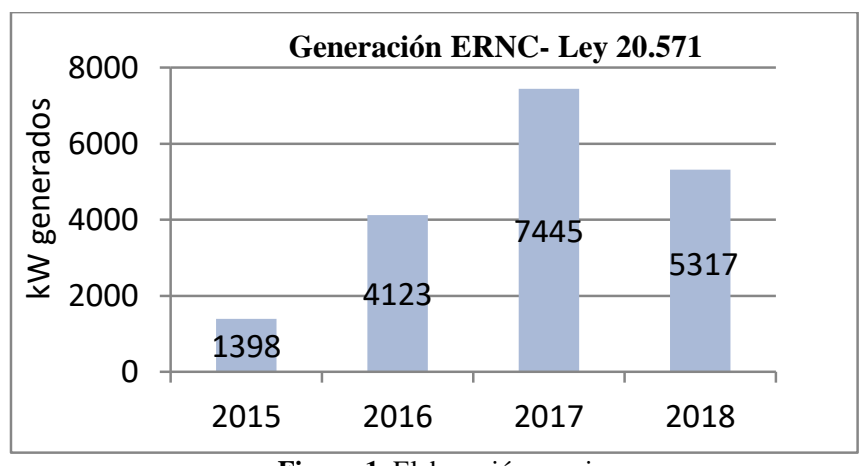

Figura 1. Elaboración propia

En la Tabla 1, podemos observar que la energía solar destaca entre sus pares, donde la potencia instalada a través de energía solar equivale al 99, $4 \%$ de potencia total instalada desde el año 2015 a la fecha, según datos recogidos de la página web www.energiaabierta.cl, del Ministerio de Energía de Chile, publicada en el año 2018 .
Tabla 1. Instalaciones de ERNC acogidas a ley 20.571

\begin{tabular}{lccc}
\hline \multicolumn{1}{c}{ Fuente Energía } & Cantidad & $\begin{array}{c}\text { Potencia } \\
\text { Instalada (kW) }\end{array}$ & $\%$ \\
\hline Biomasa & 2 & 60 & $0,33 \%$ \\
Cogeneración & 1 & 50 & $0,27 \%$ \\
Eficiente & 2 & & \\
Hidráulica & 2758 & 18.171 & $99,01 \%$ \\
Solar & $\mathbf{2 7 6 3}$ & $\mathbf{1 8 . 2 8 3}$ & $\mathbf{1 0 0 \%}$ \\
\hline Total & & & \\
\hline
\end{tabular}

Fuente: elaboración propia

\subsection{Una mirada desde la sustentabilidad ambiental}

En los últimos años, la literatura reconoce las ventajas de la implementación de fuentes energéticas renovables en la reducción de impactos ambientales y especialmente mejora en la soberanía energética con la disminución de la dependencia energética y la diversificación de la matriz energética, pero especialmente para justificar este tipo de inversiones, se hace necesario la cuantificación de estos aspectos y de una evaluación costo beneficio para determinar la conveniencia de la inversión (Cruz et al., 20016), como la ausencia de la contabilización de las externalidades en el análisis costo beneficio de estas iniciativas (Painuly, 2001).

Una de las ventajas está dada por su contribución a la reducción de emisiones de Gases de Efecto Invernadero (GEI) y otros contaminantes que proviene del uso de combustibles fósiles que se usan generalmente en las industrias, como el petróleo, el carbón o el gas natural. Siendo el Protocolo de Kyoto donde se sienta las bases de la arquitectura del mercado de carbono, donde se transa los llamados Certificados de Emisiones Reducidas, (CERs), o "bonos de carbono" (Posso, Acevedo \& Hernández, 2014), donde cada uno representa el derecho a emitir una tonelada de dióxido de carbono (tCO2), equivalente a una estimación monetaria del carbono a partir de los beneficios de las reducciones de gases de efecto invernadero. En general, aquellas empresas que reduzcan sus emisiones contaminantes por debajo de su cupo pueden vender sus bonos, los cuales pueden ser adquiridos por aquellas empresas que necesiten reducir sus emisiones para cumplir con el cupo que tienen asignado, y cuya reconversión tecnológica es más costosa que la compra de bonos.

Amorós y Micaló (2005) establecen que existen diferentes métodos de valoración para fijar un valor monetario al carbono, los cuales se pueden clasificar en tres grupos: métodos basados en los costes de los daños evitados (Method of Avoided Damages), métodos basados en los costes de reducción de las fuentes de emisiones de carbono (Mitigation Option Approach), que tratan de aproximar el valor del carbono mediante el coste de reducir las emisiones de $\mathrm{CO} 2$ que se liberan a la atmósfera y los basados en los costes de incrementar la cantidad de carbono almacenada en el sistema (Enhanced Sequestration Option o Sink option).

Para el caso chileno, Rivas \& Cardemil (2015) analizan evolutivamente distintas investigaciones del mercado del carbono en inversiones energías fotovoltaicas y especialmente las características en la fijación de las emisiones y del precio de los bonos. Lo que es evidente son las condiciones geográficas y climáticas en el recurso solar chileno y las perspectivas para la industria fotovoltaica solar el futuro despliegue de tecnología fotovoltaica en este país (Zurita, et 
al, 2018). Coincidentemente en un trabajo reciente de Simsek et al, (2018), se establecen las favorables condiciones de Chile para proyectos de energía solar concentrada especialmente en el norte, haciendo hincapié que los proyectos de energía solar térmica necesitan algunos mecanismos de apoyo y analiza mejores combinaciones de incentivos solares y parámetros financieros para un mejor desempeño del sector.

Ahora bien, a pesar de esta búsqueda de valorizar monetariamente los impactos ambientales, Müller-Steinhagen, \& Nitsch (2005) señalan que las implicaciones económicas son de amplio espectro ocultando incluso las de tipo social y por ello, todo estudio integral de las mismas amerita un enfoque multidimensional, garantizando la sustentabilidad del mismo al satisfacer el criterio de sustentabilidad basado en la compatibilidad ambiental y un uso más racional y eficiente de las fuentes de energía.

En este sentido y tomando en cuenta que el análisis se concentra en proyectos de menor escala como son de generación distribuida, surge la pregunta si ¿es económicamente rentable implementar este tipo de fuentes de generación?, para dar respuesta a esta interrogante, se ha tomado una evaluación privada y otra con la incorporación de la valoración económica ambiental. Como caso de estudio se utiliza las instalaciones fotovoltaicas existentes en la inter comuna Chillán-Chillán Viejo, que se encuentren válidamente inscritas, de acuerdo con lo establecido en la Ley 20.571. Para ello se ha hecho un levantamiento de las instalaciones existentes, analizando las variables de costo de implementación, consumo antes y después de la instalación fotovoltaica y generación de energía eléctrica de estas instalaciones, que se desarrollan a lo largo de este artículo.

\section{METODOLOGÍA}

La investigación analiza ex post la rentabilidad bajo un enfoque privado y social de las instalaciones fotovoltaicas existentes en la inter comuna de Chillán - Chillán Viejo, que se encuentren válidamente inscritas, de acuerdo con lo establecido en la Ley 20.571, que permite inyectar excedentes de energía eléctrica a las redes de distribución pública. Para lo cual se realizó un estudio de caso, utilizando la metodología de evaluación financiera de proyectos de inversión (Sapag, 2007), analizando las variables de costo inicial, ahorro de energía e inyección de excedentes a la red de distribución bajo el enfoque privado e incorporando una simulación de los beneficios por emisión de bonos de carbono como compensación de las fuentes limpias en el caso del enfoque social. Dado que la propiedad de las unidades de estudio es heterogénea (personas y empresas), existen regímenes tributarios diversos, no identificándose beneficios tributarios directos a la inversión y tampoco se incorporaron al modelo financiero general. La información se obtuvo de organismos gubernamentales y de las empresas privadas de distribución de energía eléctrica presentes en la zona.

Se utilizó como base fundamental parámetros esenciales para la viabilidad económica de la irradiación fotovoltaica y las tarifas de electricidad locales, en una adaptación a partir de la metodología de Mitscher \& Rüther (2012), quienes analizaron la competitividad económica de la generación solar fotovoltaica distribuida conectada a la red a través de instalaciones en techos a pequeña escala en cinco capitales estatales brasileñas, calculando los valores actuales netos (VAN) de cada sistema de producción fotovoltaica, modelando el flujo de ingresos a partir de la venta de electricidad fotovoltaica a las tarifas de compra de las compañías de electricidad locales y el ahorro por el autoconsumo a la tarifa residencial, asumiendo la medición neta. Se expone su formulación en la ecuación (1):

$$
V A N=I_{0}+\sum_{t=1}^{n} \frac{F_{t}}{(1+r)^{t}}
$$

Siendo $I_{0}$ la inversión inicial en el periodo cero; $F_{t}$ el flujo (ingresos - costo) en cada periodo $\mathrm{t}$; $\mathrm{n}$ el número de periodos de evaluación (horizonte del proyecto, se excluye el periodo cero); y $r$ como la tasa de descuento. A su vez para el enfoque de valoración económica ambiental, se agregan ingresos extraordinarios al flujo, simulando la obtención de la venta de "bonos de carbono" a precio de mercado, como compensación de las emisiones reducidas de $\mathrm{CO} 2$.

Para recabar la información, se solicitó al Departamento de ERNC de la Superintendencia de Electricidad y Combustibles (SEC), la base de datos de las instalaciones fotovoltaicas existentes en la intercomuna de Chillán - Chillán Viejo, con un total de 24 instalaciones válidamente inscritas y certificadas. Posteriormente, se solicitó a las empresas de distribución de energía eléctrica locales, los registros de los consumos de energía de estos servicios desde el año 2010 a la fecha, que muestra los consumos mensuales antes y después de la implementadas las instalaciones fotovoltaicas, con el objetivo de realizar la comparación de dos periodos estacionales que comprenden los mismos meses calendarios anterior y posterior a la instalación de los PFV.

De las 24 instalaciones fotovoltaicas antes mencionadas, podemos señalar que tres se encuentran recientemente conectadas y cuatro aún sin conexión a la red pública, por lo que estas siete instalaciones fueron excluidas por no tener historia y un registro de generación de energía eléctrica a través de PFV, datos necesarios para realizar el análisis financiero. Con las 17 instalaciones fotovoltaicas vigentes, foco de este estudio, se analizó consumos, ahorros y generación de energía eléctrica, este análisis consistió en tomar un periodo estacional regular antes de la implementación de la instalación fotovoltaica y obtener un consumo promedio mensual del servicio, considerando para el mismo periodo estacional una vez conectada la instalación fotovoltaica a la red de distribución y de esta forma obtener un consumo promedio mensual del servicio. De la comparación de estos periodos se obtuvo el ahorro de energía eléctrica, valorizado a precio de compra que se le cancela a la empresa eléctrica, que en el caso CGE corresponde a CLP\$125 del kWh mensual y CLP\$126 del kWh mensual para COPELEC.

Posteriormente, se realizó el cálculo de la inyección promedio mensual de energía, que suministra el servicio a la red de la empresa de distribución, registro obtenido a través de la lectura de los medidores electrónicos, el cual se encuentra separado de 
la inyección al sistema y del consumo de energía. Esta inyección mensual de energía se mide en $\mathrm{kWh}$ mes y se valoriza a CLP\$78. Esta tarifa corresponde a los llamados "clientes regulados", cuya potencia conectada es inferior a 500 $\mathrm{kW}$. Los precios se fijan mediante los decretos de "precio de nudo" que emite la Comisión Nacional de Energía (CNE), perteneciente al Ministerio de Minería de Chile.

Para realizar el estudio de factibilidad económica de cada instalación, se consideró la recomendación de GIZ (2015), que propone evaluar los sistemas fotovoltaicos con una vida útil de 25 años, desde una perspectiva técnico-económica. Por lo cual se proyectó un flujo de caja con un horizonte de evaluación a 10 años, concordante con los contratos con las empresas compradoras de energías de Chile, considerando un valor desecho económico por los restantes años que faltan en el último periodo $(\mathrm{t}=10)$. Para calcular el Valor Actual Neto (VAN) y la Tasa Interna de Retorno (TIR), se consideró la tasa de descuento propuesta por el Ministerio de Energía del Gobierno de Chile (2017) de $8,74 \%$ para proyectos de tecnología solar fotovoltaica.

A partir de los resultados obtenidos, en términos de rentabilidad de rentabilidad privada, se realizó un análisis de sensibilidad sobre esta variable inversión inicial y flujos anuales, para ver de qué manera esta influye en la rentabilidad del proyecto.

En el caso de la valoración económica ambiental, se utiliza el estudio técnico anterior como base, cuantificando el monto de las inversiones, beneficios económicos y costos de operación (Sapag, 2007). El flujo en cada periodo se ve compensado con la incorporación de beneficios económicos provenientes de la simulación de ventas de "bonos de carbono", como compensación y reconocimiento en la disminución de emisiones de gases a la atmosfera, para los cual es necesario un análisis de reducción de emisiones que permita el cálculo de huella de carbono por consumo eléctrico. En términos simples, la huella de carbono es el registro de gases de efecto invernadero (GEI) que empresas e individuos lanzan al planeta, ya sea directa o indirectamente. En Chile, el factor emisión de GEI utilizado para el territorio analizado, es el correspondiente al Sistema Interconectado Central (SIC) para el año 2016 equivalente a 0,397tCO2/MWh. El trabajo no presenta por sí mismo cálculos del factor de emisión, sino más bien, el cálculo de emisiones del caso estudiado se simuló en una empresa dedicada al asesoramiento técnico en la compraventa de derechos de emisión por cuenta propia en la página

CEROCO2 (https://www.ceroco2.org/calculadoras/electric), al cual se ingresaron los datos de las instalaciones FV con su respectiva capacidad de $\mathrm{kW}$ generados.

A su vez, para una empresa que reduce sus emisiones es posible estimar el valor económico de esta reducción de emisiones (Rivas \& Cardemil, 2015), teniendo la opción de certificar los gases que no lanzó al medio ambiente y convertirlos en bonos de carbono o en Certificados de Emisiones Reducidas (CER). En Chile, según la Bolsa de Clima de Santiago SCX (http://www.scX.cl) un bono de carbono o CER, es decir, una tonelada de CO2 que se deja de emitir a la atmósfera, se transaba en un precio promedio de US\$ 12 (Rivas \& Cardemil, 2015). Otro escenario es simular al "impuesto verde" que impone un pago de 5 US\$ por cada tonelada de $\mathrm{CO} 2$ emitida por el Estado en Chile. Es importante precisar que los ingresos por venta de bonos de carbono son calculados solo para un año y replicados en los años siguientes, con lo cual no se considera las posibles variaciones del factor de emisión en la matriz energética.

\section{RESULTADOS}

De las 24 instalaciones certificadas por la SEC, 33\% de las instalaciones corresponden al territorio de la empresa eléctrica COPELEC y un $67 \%$ la empresa CGE. Asimismo, respecto al tipo de proyecto, un $8,3 \%$ de total de instalaciones corresponde al sector agrícola, un $4,2 \%$ al sector industrial, un $8,3 \%$ al comercio y un $79,2 \%$ al sector habitacional.

Ahora bien, al revisar la inversión por sector en kilowatts (potencial), la distribución porcentual cambia. El 22,5\% de inversión la concentra el sector agrícola, $29 \%$ el comercio, $14,2 \%$ en el sector industrial, en tanto el sector habitacional, el que posee mayor número de instalaciones (19), llega en total a un $34,3 \%$ de inversión en kilowatts. En coherencia con los datos anteriores, en términos económicos la mayor inversión se ha realizado en el sector habitacional que representa el $35,3 \%$ del total ejecutado en el periodo, en seguido por los sectores comercio con un $27,6 \%$, agrícola con un $23,7 \%$ y por último el industrial que concentra una inversión monetaria del $13,4 \%$. Se destaca que el promedio de inversión para producir un kilowatt es de CLP\$ 1.811.259, equivalente a US\$ $2.703,42^{1}$. El mayor costo de instalación es en el sector agrícola con una inversión promedio por kilowatts de CLP\$ 1.908 .932 (US\$ $2.849,2$ ), siendo un 5,4\% mayor al promedio total. En tanto, el del menor costo se ve reflejado en el sector industrial con CLP\$ 1.719.359 (US\$ 2.566,2), lo cual significa un $5,1 \%$ menos que el promedio.

Sin embargo, y en coherencia con lo señalado en la metodología de las 24 instalaciones fotovoltaicas antes mencionadas, solo 17 de estas instalaciones fueron incluidas en el estudio financiero (Tabla 2), excluyendo 7 instalaciones; 3 de ellas recientemente conectadas y 4 sin conexión al sistema de distribución, no existiendo aun registro de la generación de energía eléctrica determinante que fije un comportamiento, dato necesario para obtener una generación promedio de la instalación.

Cabe destacar que de 17 instalaciones en operación (Tabla $\mathrm{N}^{\circ}$ 2), la mayor cantidad se concentra en el sector habitacional (13), asimismo es la que presenta el mayor costo de inversión por kilowatts con CLP\$ 1.884.138 (USD\$ 2.812,1), siendo un $4,7 \%$ más que el promedio general (CLP\$ 1.800.123 = USD\$ $2.686,75)$. En tanto, el sector industrial presenta el menor el costo de inversión por kilowatts alcanzando los CLP\$ 1.719.359 (USD\$ 2.566,2), un 4,5\% menos que el promedio.

\footnotetext{
${ }^{2}$ Tipo de Cambio CLP\$ $670=1$ U\$S americano
} 
Tabla 2. Instalaciones activas y con venta de energía

\begin{tabular}{llcrr}
\multicolumn{1}{c}{ Sector } & $\mathbf{N}^{\circ}$ & $\begin{array}{c}\text { Potencia } \\
(\mathbf{k W})\end{array}$ & $\begin{array}{c}\text { Inversión en } \\
\text { CLP\$ }\end{array}$ & $\begin{array}{c}\text { Costo inversión } \\
\text { por kW en CLP\$ }\end{array}$ \\
\hline Agrícola & 1 & 29,40 & 54.589 .498 & 1.856 .786 \\
Comercio & 2 & 41,00 & 70.676 .965 & 1.723 .828 \\
Industrial & 1 & 20,00 & 34.387 .186 & 1.719 .359 \\
Habitacional & 13 & 36,63 & 69.015 .959 & 1.884 .138 \\
\hline Total & $\mathbf{1 7}$ & $\mathbf{1 2 7 , 0 3}$ & $\mathbf{2 2 8 . 6 6 9 . 6 0 8}$ & $\mathbf{1 . 8 0 0 . 1 2 3}$ \\
\hline Fuente: elaboración propia
\end{tabular}

Fuente: elaboración propia

Al realizar la evaluación económica del proyecto, en la Tabla 3 se muestra el comportamiento total de las 17 instalaciones en operación y por sector, donde se puede observar que el ingreso económico se tributa por dos vías, una a través del ahorro mensual del cliente obtenido del menor consumo de electricidad producto de la instalación de los paneles fotovoltaico y la otra vía es por la inyección de los excedentes de energía eléctrica generados por el cliente hacia la red de la empresa eléctrica. Estas dos vías de ingresos se suman y se consolidan en un ingreso anual por sector. La información respecto a los consumos promedios mensuales antes y posterior a la conexión de las instalaciones fotovoltaicas se obtuvieron de las empresas eléctricas que suministran energía a los clientes y que a la vez pagan los excedentes de energía inyectados a la red, reconociendo el promedio móvil por cada sector.

Tabla 3. Proyecciones económicas de costos e ingresos

\begin{tabular}{llllll}
\hline \multirow{2}{*}{ Sector } & $\begin{array}{l}\text { Ahorro } \\
\text { energía } \\
\text { (kWh/mes) }\end{array}$ & $\begin{array}{l}\text { Ahorro } \\
\text { mes } \\
\text { (CLP\$) }\end{array}$ & $\begin{array}{l}\text { Energía } \\
\text { inyectada } \\
\text { (kW/h } \\
\text { mes) }\end{array}$ & $\begin{array}{l}\text { Ingreso } \\
\text { energía } \\
\text { vendida } \\
\text { (CLP\$) }\end{array}$ & $\begin{array}{l}\text { Ingreso } \\
\text { promedio } \\
\text { año } \\
\text { (CLP\$) }\end{array}$ \\
\hline Agrícola & $-836,0$ & 0 & $1.610,0$ & 125.580 & 1.506 .960 \\
Comercio & $-177,0$ & 0 & $2.917,0$ & 227.526 & 2.730 .312 \\
Industrial & 236,0 & 29.500 & 823,0 & 93.694 & 1.124 .328 \\
Habitacional & 827,0 & 126.250 & $2.801,0$ & 344.728 & 4.136 .736 \\
\hline Suma & $\mathbf{5 0 , 0}$ & $\mathbf{1 5 5 . 7 5 0}$ & $\mathbf{8 . 1 5 1 , 0}$ & $\mathbf{7 9 1 . 5 2 8}$ & $\mathbf{9 . 4 9 8 . 3 3 6}$ \\
\hline
\end{tabular}

Fuente: elaboración propia

Al analizar cada sector y a su vez las dos vías de ingreso (ahorro e inyección al sistema), se puede apreciar que el sector agrícola y comercio, no tienen ingresos por concepto de ahorros de energía, debido a que posterior a la conexión de las instalaciones fotovoltaicas, ambos sectores inclusive evidenciaron un aumento en sus consumos mensuales de energía. Sin embargo, se puede observar que la inyección mensual de energía al sistema es alto respecto a los otros sectores del estudio, con un promedio de $1.610 \mathrm{kWh} / \mathrm{mes}$ para el sector agrícola y $2.917 \mathrm{kWh} / \mathrm{mes}$ para el sector comercio, esto se puede deber a que la potencia instalada es superior respecto a los otros sectores. Ahora bien, para el sector industrial y habitacional, se aprecia que existe ingreso por concepto de ahorro y de inyección de excedentes de energía, pero ambos ingresos son bastante marginales respecto a las inversiones iniciales. Para el sector residencial podemos observar que existen ingresos por ambas vías, destacando la inyección a la red $(2.801,0 \mathrm{kWh} / \mathrm{mes})$ por sobre el ahorro mensual (827,0 $\mathrm{kWh} / \mathrm{mes})$, además se aprecia que el consolidado anual corresponde al mayor ingreso registrado de todos los sectores de estudio, a pesar de ser el sector con la menor potencia instalada por cliente.

\subsection{Rentabilidad Privada}

Con los datos entregados por los actores involucrados, correspondiente a las 17 instalaciones en estudio, en la Tabla 4 , se construyó la proyección de rentabilidad asociada a cada sector y en su totalidad.

De acuerdo con lo observado en la Tabla 4, el Valor Actual Neto (VAN) resulta negativo para todos los sectores, es decir, bajo estas condiciones se puede inferir que no es rentable realizar este tipo de proyectos en forma privada, debido a que los flujos de ingreso anuales obtenidos son muy bajos respecto a la inversión inicial realizada, teniendo en consideración que los costos de la inversión inicial son valores de mercado.

\begin{tabular}{|c|c|c|c|c|}
\hline Sector & $\begin{array}{l}\text { Flujo } \\
\text { periodo } \\
\text { CLP\$ } \\
(t=1 \ldots t=10)\end{array}$ & $\begin{array}{l}\text { Valor } \\
\text { Desecho } \\
\text { Económico } \\
\text { t=10 CLP\$ }\end{array}$ & VAN (CLP\$) & TIR \\
\hline Agrícola & 1.506 .960 & 17.242 .105 & - 37.347.393 & $-6,2 \%$ \\
\hline Comercio & 2.730 .312 & 31.239 .268 & - 39.437.697 & $-2,3 \%$ \\
\hline Industrial & 1.124 .328 & 12.864 .165 & -21.523 .021 & $-4,3 \%$ \\
\hline Habitacional & 4.136 .736 & 47.331 .076 & -21.684 .883 & $3,3 \%$ \\
\hline Suma & 9.498.336 & 108.676 .613 & - 119.992.995 & $-1,4 \%$ \\
\hline
\end{tabular}

Fuente: elaboración propia

\subsection{Sensibilización de los proyectos con subsidio a la inversión inicial}

Con los datos entregados por los propios actores (17) y en coherencia con los resultados anteriores que dan un resultado económico adverso, en la Tabla 5 se simuló el subsidio necesario a la inversión inicial que permitiría la rentabilidad mínima exigida $(8,74 \%)$, pudiendo establecer que es necesario un subsidio base del $52,5 \%$ promedio, siendo la menor necesidad en el sector habitacional con un $31,4 \%$ y el de mayor ayuda el sector agrícola con un $68,4 \%$ de subsidio directo.

Tabla 5. Sensibilización con subsidio a la inversión Inicial

\begin{tabular}{|c|c|c|c|}
\hline Sector & $\begin{array}{l}\text { Inversión inicial } \\
\text { CLP\$ }\end{array}$ & Subsidio CLP\$ & $\%$ Subsidio \\
\hline Agrícola & 54.589 .498 & 37.347 .393 & $68,4 \%$ \\
\hline Comercio & 70.676 .965 & 39.437 .697 & $55,8 \%$ \\
\hline Industrial & 34.387 .186 & 21.523 .021 & $62,6 \%$ \\
\hline Habitacional & 69.015 .959 & 21.684 .883 & $31,4 \%$ \\
\hline Suma & 228.669 .608 & 119.992 .995 & $52,5 \%$ \\
\hline
\end{tabular}

Fuente: elaboración propia

\subsection{Sensibilización de los proyectos con subsidio a la tarifa mensual}

Alternativamente al escenario anterior de subsidio a la inversión, se estimó un escenario que subsidiara los ingresos anuales, encontrando para la rentabilidad mínima exigida por los proyectos que es necesario un subsidio del 110,4\%. Coincidentemente con la evaluación anterior, es en el sector habitacional donde se necesita menos subsidio a los precios con un $45,8 \%$. En tanto el sector agrícola, bajo estas condiciones, necesitaría un subsidio anual del $216,6 \%$ al precio. Véase Tabla 6. 
Tabla 6. Proyección de rentabilidad con subsidio al precio

\begin{tabular}{llll}
\hline \multicolumn{1}{c}{ Sector } & $\begin{array}{c}\text { Flujo por periodo } \\
\text { CLP\$ } \\
\text { (años t= 1...t=10) }\end{array}$ & $\begin{array}{c}\text { Subsidio estimado al } \\
\text { flujo anual CLP\$ }\end{array}$ & $\begin{array}{c}\% \\
\text { Subsidio }\end{array}$ \\
\hline Agrícola & 1.506 .960 & 3.264 .162 & $216,6 \%$ \\
Comercio & 2.730 .312 & 3.446 .855 & $126,2 \%$ \\
Industrial & 1.124 .328 & 1.881 .112 & $167,3 \%$ \\
Habitacional & 4.136 .736 & 1.895 .259 & $45,8 \%$ \\
\hline Suma & $\mathbf{9 . 4 9 8 . 3 3 6}$ & $\mathbf{1 0 . 4 8 7 . 3 8 8}$ & $\mathbf{1 1 0 , 4 \%}$ \\
\hline
\end{tabular}

Fuente: elaboración propia

\subsection{Rentabilidad con valoración económica ambiental}

Tal como se señaló en la metodología, teniendo como base los datos base de la evaluación privada, se agregaron al flujo económico anual, la simulación de ingresos por "bonos de carbono" con una emisión de gases (GEI), equivalente para la generación anual (118.639kW) considerando la conversión de kW a MW, obtenemos una emisión equivalente de 47,10 tCO2 por MW, a US\$ 12 por tonelada En la Tabla 7, se construyó la proyección asociada a cada sector y en su totalidad.

\begin{tabular}{|c|c|c|c|c|c|}
\hline Sector & tCO2 & $\begin{array}{l}\text { Flujo } \\
\text { Privado } \\
\text { CLP\$ } \\
(t= \\
1 \ldots t=10)\end{array}$ & $\begin{array}{l}\text { Flujo } \\
\text { Bonos de } \\
\text { Carbono } \\
\text { CLP\$ } \\
(t= \\
1 . . t=10) \\
\end{array}$ & $\begin{array}{l}\text { VAN Social } \\
\text { (CLP\$) }\end{array}$ & $\begin{array}{l}\text { TIR } \\
\text { Social }\end{array}$ \\
\hline Agrícola & 10,90 & 1.506 .960 & 87.636 & -36.344 .693 & $-5,6 \%$ \\
\hline Comercio & 15,20 & 2.730 .312 & 122.208 & - 38.039.436 & $-1,8 \%$ \\
\hline Industrial & 7,42 & 1.124 .328 & 59.657 & -20.840 .449 & $-3,7 \%$ \\
\hline Habitacional & 13,58 & 4.136 .736 & 109.183 & - 20.435.648 & $3,6 \%$ \\
\hline Suma & 47,10 & 9.498 .336 & 378.684 & $\begin{array}{l}- \\
115.660 .226\end{array}$ & $-1,0 \%$ \\
\hline
\end{tabular}

Fuente: elaboración propia

De acuerdo con lo observado en la Tabla 7, el efecto de incorporar el "Bono de Carbono" a la simulación de flujos de cada periodo, el Valor Actual Neto (VAN) para una valoración ambiental no mejoró de manera significa. Ahora bien, al sensibilizar la variable precio por tonelada de carbono resulta bajo como valor compensatorio.

\section{DISCUSIÓN Y ANÁLISIS DE LOS RESULTADOS}

Los resultados económicos dan cuenta que los proyectos analizados para este estudio caso de menor escala, desde la perspectiva privada, no son rentables en ninguno de los sectores estudiados, obteniendo un VAN negativo y por ende, una TIR no aceptable. Este deficiente desempeño económico puede ser explicado por varios factores, por ejemplo, un alto costo de la inversión inicial para la generación de energía fotovoltaica que aún es difícil recuperar con los flujos que se generan y aunque el costo ha disminuido en los últimos años, todavía es necesario un menor valor para hacer más atractivos este tipo de proyectos y por ende, se necesita algún tipo de incentivo (Correa, Marulanda \& Panesso, 2016). Asimismo, se puede pensar que los proyectos en ejecución, tienen un problema de eficiencia técnica que se traduce en una baja generación eléctrica y por lo tanto, no han alcanzado el nivel de desempeño con el cual fueron diseñados, atribuibles a temas técnicos como ubicación, falta de mantención, deficiencias en la operación, entre otros.
Además, se observó un fenómeno que afectó a todas las instalaciones en forma transversal, que fue el aumento en el consumo de energía, posterior a la conexión de la instalación fotovoltaica, este cambio de comportamiento puede ser base para un estudio posterior, pero una hipótesis que se puede establecer es la interpretación de los clientes, que el hecho de generar su propia energía da cierta libertad de consumo y se dejan de lado las auto restricciones previamente existentes.

De acuerdo al estado de desarrollo de los proyectos estudiados, es evidente que es necesario un subsidio en la inversión inicial o en los precios (tarifa de transferencia), tal como se realiza en otras latitudes, especialmente en Europa (Durán, 2013). Existiendo distintos incentivos y/o mecanismos de promoción, tales como el "Fett in Tarif "(fijación de tarifa), compra de "certificados verdes", sistemas de incentivos a la producción de ERNC, entre otros. Lo que se hace evidente, es que el tipo y nivel de incentivos deben determinarse cuidadosamente en los procesos de formulación de políticas públicas que permitan promover de manera efectiva los sistemas fotovoltaicos (Zhang, Knizley \& Cho, 2017), especialmente con los hallazgos encontrados donde el sector habitacional es el que menos subsidio necesitaría. En cambio, el sector agrícola supone un mayor apoyo económico; ya que debe considerar elementos constructivos para soportar la instalación fotovoltaica y que se evidencian en el mayor costo promedio de inversión por kilowatts entre los distintos sectores estudiados, coincidente con los estudios de González (2014) donde establece que el costo total de instalación puede alcanzar hasta el $40 \%$.

También se puede establecer una crítica al precio de compra del kilowatts (tarifa) por parte de las empresas eléctricas, ya que se puede inferir e asimetrías de información, ya que no se conocen las estructuras de costos reales, significando un desincentivo a generar electricidad limpia a menor escala.

Los resultados para este estudio de caso, hacen suponer que se mantiene la premisa observada en estudios anteriores de otras latitudes, donde se establece que los sistemas fotovoltaicos poseen una mayor inversión inicial pero sus costos de producción energética unitaria son menores a otras fuentes energéticas, por lo tanto, generan una mayor rentabilidad a mediano y largo plazo (Mahmoud \& Ibrik, 2006).

\section{CONCLUSIONES}

Se plantea que la energía solar fotovoltaica es una buena alternativa a los problemas de generación del país, generando beneficios de operación y confiabilidad de la red, pero más aún sus externalidades positivas al medio ambiente y su impacto social. Se identifican distintos actores en la producción fotovoltaica, grandes y pequeños. En estos últimos, los de generación distribuida son clave para la competitividad del sector. Sin embargo, al evaluar económicamente estos proyectos desde una perspectiva privada, es decir sin presencia del Estado, los proyectos acogidos a la Ley 20.571 muestran para el caso en estudio que no son rentables.

Los resultados obtenidos muestran, a partir de su actual condición de operación, que no son atractivos 
económicamente para los inversores. Estos resultados son coincidentes con los estudios de Recalde (2017) para Argentina, quien concluye que no obstante se reconoce la importancia de promover las energías renovables y su potencial para el impacto ambiental, aún enfrentan el problema de mayores costos y menor rentabilidad relativa que las tecnologías convencionales maduras, lo que ha dificultado su difusión en todo el mundo. Por ello si el objetivo del gobierno en Chile es promover la generación distribuida, es preciso concebir estrategias necesarias para generar rentabilidad de este tipo de proyectos. Las fórmulas elegidas pueden ser variadas, tal como el subsidio a la instalación o a la tarifa. Lo que no está en discusión, es que el gobierno pretenda incentivar la generación distribuida y a la vez deje al mercado como ente regulador, sabiendo que los resultados no pueden ser otros que una ambigüedad. Sabemos que Chile es un país inmerso en el sistema de libre mercado, donde el Estado interviene lo menos posible en los mercados, pero si se desea promover esta industria, se hace necesario replicar buenos ejemplos como España y Alemania, quienes incentivan la generación distribuida por medio de estrategias tales como la fijación de tarifas o subsidios a la inversión (Jiménez, 2011), especialmente en sectores residenciales. Sin la intervención del Estado apoyando económicamente esta incipiente industria, todo quedará en una promesa que apuntaba a incentivar una nueva forma de generar energía a pequeña escala y que desarrollaría tras ella una nueva industria movida por emprendedores.

Una última preocupación viene dada por las señales de la autoridad de gobierno que está promoviendo un proyecto de ley que modifica la actual Ley 20.571 (Ministerio de Energía del Gobierno de Chile, 2018), dentro de los alcances de esta modificación, está el limitar el pago de los excedentes inyectados, acumulándolos en la cuenta y descontando del consumo del cliente, así solo se cancelarían las inyecciones de aquellos clientes cuyos excedentes superen los consumos generados en un periodo de tiempo equivalente a cinco años. Lo anterior, puede provocar un desincentivo en la inversión de pequeños fuentes de generación, que puede llevar a destruir esta insipiente industria.

A pesar de que las evaluaciones ex post de las casos estudiados de generación distribuida no son los esperados desde la perspectiva privada e incluyendo la valoración económica ambiental. Es importante enfatizar, de que la región necesita implementar proyectos en base a energías renovables, considerando por un lado políticas de incentivo estatal directo a los inversionistas y posibilidades de financiamiento creciente a través de los bonos de carbono. Teniendo claro y como lo ha establecido la literatura, que a pesar de utilizar instrumentos más o menos similares los países avanzan en distintos grados debido a factores políticos, económicos e institucionales que enmarcan las decisiones de política, por lo cual es importante avanzar a ciudades más responsables con su medio ambiente, generando acciones concretas para un mayor educación, concientización ecológica, regulación de consumos, entre otras medidas.

Asimismo, es importante enfatizar las limitaciones de estudio en cuanto a plantear una perspectiva económica-financiera a un tema de alta complejidad, pudiéndose en estudios futuros incorporar efectos tributarios por tipo de inversionista o más interesante aún, comparar este modelo con el método de "costo medio de generación" para cada proyecto fotovoltaico, conocido como LCOE (levelized costo of energy), tanto a nivel local o regional.

\section{REFERENCIAS}

Ackermann, T., Andersson, G. \& Söder, L. (2001). Distributed generation: a definition. Electric Power System Research. 57, 195-204.

Amorós, J. M., \& Micaló, P. R. (2005). El valor de la fijación de carbono en los programas de forestación. Boletín económico de ICE, (2834), 13-28.

Azqueta Oyarzun, D. (1994). Valoración económica de la calidad ambiental. McGraw-Hill, Madrid.

Behnke, D. R. P., Estévez, G. J., \& Arias, I. A. (2009). Las Energías Renovables No Convencionales En El Mercado Eléctrico Chileno. Proyecto Energías Renovables No Convencionales (CNE/GTZ), 124.

Bermejo, R. (2013). Ciudades Postcarbono y Transición Energética. Revista de Economía Crítica, 16, 215-243.

Correa F., C.A.; Marulanda G., G.A. \& Panesso H., A.F. (2016). Impacto de la penetración de la energía solar fotovoltaica en sistemas de distribución: estudio bajo supuestos del contexto colombiano. Revista Tecnura, 20(50), 85-95. doi: 10.14483/udistrital.jour.tecnura.2016.4.a06

Cruz, I., Muñóz, M., Sauad, J., Altobelli1, F. \& Condorí, M. (2016). Evaluación financiera y económica de la sustitución parcial de fuentes convencionales por energía solar en un proceso industrial. Estudio de caso: la inclusión de energía solar en el Curado de tabaco. Acta de la XXXIX Reunión de Trabajo de la Asociación Argentina de Energías Renovables y Medio Ambiente. 4, 12115-12125.

Dalmazzo-Bermejo, E., Valenzuela-Klagges, B. \& EspinozaBrito, L. (2017). Producción de energía renovable no tradicional en América Latina: economía y políticas públicas. Apuntes, 44(81), 67-87. https://dx.doi.org/10.21678/apuntes.81.806

Durán, R. (2013). Mecanismos de promoción de energías renovables, comparación de sus aplicaciones en Europa, Latinoamérica y argentina de acuerdo a sus características político económicas. Avances en Energías Renovables y Medio Ambiente, Actas ASADES 2013. 17, 43-49.

GIZ (2015). Modelo de Negocios ESCO para la Generación de Energía Fotovoltaica. Deutsche Gesellschaft für Internationale Zusammenarbeit (GIZ) GmbH. [En línea] Available at: www.4echile.cl/asset/documents/688 
González, D. (2014). Tesis Doctoral, Modelo de innovación y optimización de la energía solar en España. La aplicación de las tecnologías disponibles para el aprovechamiento de recursos, comercialización y beneficios de la energía solar, Universidad Rey Juan Carlos, España, 212 pág.

Jacobs, D., Marzolf, N., Paredes, J. R., Rickerson, W., Flynn, H., Becker-Birck, C., \& Solano-Peralta, M. (2013). Analysis of renewable energy incentives in the Latin America and Caribbean region: The feed-in tariff case. Energy Policy, 60, 601-610. doi:10.1016/j.enpol.2012.09.024.

Jager-Waldau, A. (2007). Photovoltaics and renewable energies in Europe. Renewable and Sustainable Energy Reviews. 11, 1414-1437

Jiang, L., \& O'Neill, B. C. (2004). The energy transition in rural China. International Journal of Global Energy, 21(1-2), 2-26. DOI: 10.1504/IJGEI.2004.004691

Jiménez, S. (2011). Energía Renovable No Convencional: Políticas de Promoción en Chile y el Mundo. Libertad y Desarrollo, 218, 1-60.

Mahmoud, M., \& Ibrik, I. (2006). Techno-economic feasibility of energy supply of remote villages in Palestine by PVsystems, diesel generators and electric grid. Renewable and Sustainable Energy Reviews. 10 (2), 128-138. https://doi.org/10.1016/j.rser.2004.09.001

Medina, J. (2010). La dieta de dióxido de carbono CO2. Conciencia y tecnología, 39, 50-53.

Mitscher, M. \& Rüther, R. (2012). Economic performance and policies for grid-connected residential solar photovoltaic systems in Brazil, Energy Police, 49, 688-694.

Ministerio de Economía, Fomento y Reconstrucción del Gobierno de Chile (2008). Ley 20.257. Introduce modificaciones a la ley general de servicios eléctricos respecto de la generación de energía eléctrica con fuentes de energías renovables no convencionales. Disponible https://www.leychile.cl/Navegar?idNorma=270212.

Ministerio de Energía del Gobierno de Chile (2012). Ley 20.571. Regula el pago de las tarifas eléctricas de las generadoras residenciales. Disponible en: https://www.leychile.cl/Navegar?idNorma=1038211.

Ministerio de Energía del Gobierno de Chile (2014). Decreto que Modifica decreto supremo $N^{o} 71$, de 2014, del Ministerio de Energía, que aprueba reglamento de la Ley $\mathrm{N}^{\mathrm{o}} 20.571$, que regula el pago de las tarifas eléctricas de las generadoras residenciales. Disponible en: http://www.minenergia.cl/archivos_bajar/consulta_ciud adana/2016/05/netmetering/consulta.pdf
Ministerio de Energía del Gobierno de Chile (2017). Informe final: Méritos económicos, riesgos y análisis de competencia en el mercado eléctrico chileno de las distintas tecnologías de generación de electricidad, ASSET, $136 \mathrm{p}$.

Ministerio de Energía del Gobierno de Chile (2018a). Modifica Decreto Supremo $N^{o} 71$, de 2014, Ministerio de Energía, Reglamento de la Ley No 20.571.

Ministerio de Energía del Gobierno de Chile (2018b). Energía 2050 política energética de Chile. Disponible en: http://www.minenergia.cl/archivos bajar/LIBROENERGIA-2050-WEB.pdf

Müller-Steinhagen, H., \& Nitsch, J. (2005). The contribution of renewable energies to a sustainable energy economy. Process Safety and Environmental Protection, 83(4), 285-297. https://doi.org/10.1205/psep.05084.

Ovalle-Cubillos, R. (2014). Sociedad fotovoltaica. Ingeniare. Revista Chilena de Ingeniería, 22(1), 4-5. DOI: 10.4067/S0718-33052014000100001

Painuly, J. P. (2001). Barriers to renewable energy penetration; a framework for analysis. Renewable energy, 24(1), 7389.

Palma, R., Jiménez, G. \& Alarcón, I. (2009). Las Energías Renovables No Convencionales En El Mercado Eléctrico Chileno. Proyecto Energías Renovables No Convencionales, 1, 1-124.

Parlamento Europeo y Consejo de la Unión Europea (2009). Directiva 2009/28/CE relativa al fomento del uso de energía procedente de fuentes renovables y por la que se modifican y se derogan las Directivas 2001/77/CE y 2003/30/CE. Diario Oficial de la Unión Europea, 140, $16-62$.

Posso, F., Acevedo, J., \& Hernández, J. (2014). El impacto económico de las energías renovables. Revista de investigación en administración e ingeniería, 2 (2), 4954.

Plá, J., Bolzi, C. \& Durán, J.C (2018). Energía Solar Fotovoltaica. Generación Distribuida conectada a la red. Ciencia e Investigación, 68(1), 51-64.

Recalde, M. (2017). La inversión en energías renovables en Argentina. Revista de Economía Institucional, 19(36), 231-254 .https://dx.doi.org/10.18601/01245996.v19n36.09

Rivas, R. T., \& Cardemil, J. M. (2015). Evaluación de la contribución de los bonos de carbono a la competitividad de las centrales solares de concentración en Chile. Ingeniare. Revista Chilena de Ingeniería, 23(4), 609621. https://dx.doi.org/10.4067/S0718- 
Rüther, R. \& Zilles, R (2011). Making the case for gridconnected photovoltaics in Brazil. Energy Policy, 39 (3), 1027-1030.

https://doi.org/10.1016/j.enpol.2010.12.021.

Salazar-Peralta, A., Pichardo-S, A. \& Pichardo-S, U. (2016). La energía solar, una alternativa para la generación de energía renovable. Revista de Investigación $y$ Desarrollo, 2(5), 11-20.

Sanz-Casado, E., Lascurain-Sánchez, M., Serrano-López, A., Larsen, B. \& Ingwersen, P. (2014). Production, consumption and research on solar energy: The Spanish and German case. Renewable Energy, 68, 733-744.

Sapag Chain, N. (2007). Proyectos de inversión: formulación y evaluación. Pearson Educación de México SA, México.

Simsek, Y., Mata-Torres, C., Guzmán, A. M., Cardemil, J. M., \& Escobar, R. (2018). Sensitivity and Effectiveness Analysis of Incentives for Concentrated Solar Power Projects in Chile. Renewable Energy. 129 (A), 214-224.

World Economic Forum (2017). Informe Energía, Disponible: https://es.weforum.org/agenda

Zhang, J., Knizley, A., \& Cho, H. (2017). Investigation of existing financial incentive policies for solar photovoltaic systems in US regions. Aims Energy, 5(6), 974-996. DOI:10.3934/energy.2017.6.974

Zurita, A., Castillejo-Cuberos, A., García, M., Mata-Torres, C., Simsek, Y., García, R., \& Escobar, R. A. (2018). State of the art and future prospects for solar PV development in Chile. Renewable and Sustainable Energy Reviews, 92, 701-727.

\section{BIOGRAFÍAS}

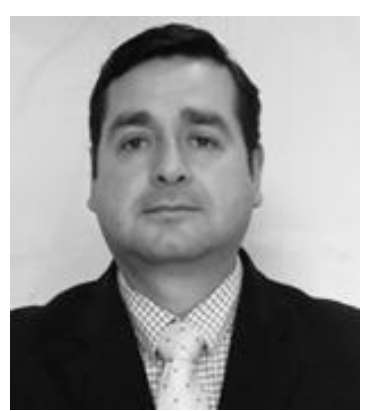

Luis Arturo Cisterna Arellano. Magíster en Gestión de Empresa de la Universidad del Bío-Bío e Ingeniero en Electricidad de la Universidad de La Frontera de Chile. Actualmente (6 años) se desempeña en la Superintendencia de Electricidad y Combustibles del Ministerio de Energía de Chile, además cuenta con 14 años de desarrollo profesional en Empresas Concesionarias de Distribución de Energía Eléctrica. ORCID: https://orcid.org/0000-0001-5793-9973

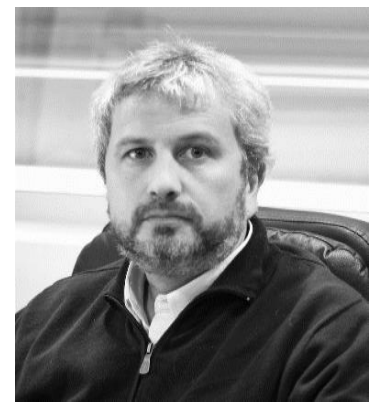

Luis Améstica-Rivas. Dr. en Administración y Dirección de Empresas, Universidad Politécnica de Cataluña, España, MBA - Magíster en Administración mención en Gestión Financiera, U. de Chile. Académico Departamento de Gestión Empresarial, Facultad de Ciencias Empresariales, Universidad del Bío-Bío, Chile. Profesor de finanzas y proyectos en distintas universidades chilenas en pre y postgrado. Ha sido gerente de finanzas y de empresas, consultor y directivo universitario. Investigador asociado grupo de investigación en Dirección Universitaria de la Universidad Politécnica de Cataluña, España. ORCID: https://orcid.org/0000-0003-0482-0287

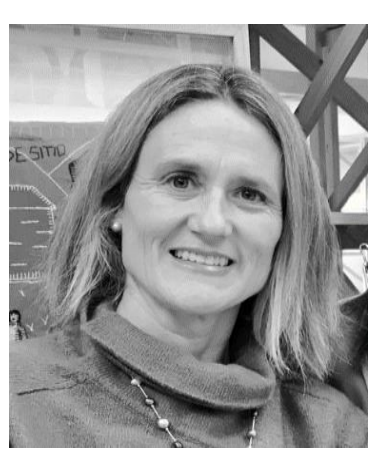

María Beatriz Piderit Moreno. Arquitecta, Magister en Ciencias Aplicadas y Doctor de la Universidad Católica de Lovaina, Bélgica. Docente de la Facultad de Arquitectura, Construcción y Diseño de la Universidad del BíoBío. Especialista del área de la Arquitectura Sustentable, ha desarrollado investigación y proyectos en el área de la Iluminación Natural, Espacios Educativos y Confort Ambiental. Directora del programa del Magister Hábitat Sustentable y Eficiencia Energética, además se desempeña como docente del Doctorado Arquitectura y Urbanismo de la Universidad del Bío-Bío. Investigadora activa del Centro de Investigación en Tecnologías de la Construcción de la Universidad, ha participado en diferentes proyectos de investigación y desarrollado publicaciones en revistas científicas del área. ORCID: https://orcid.org/00000001-9733-6608 